\title{
Expansão geográfica da leishmaniose visceral no Estado de Pernambuco
}

\author{
Geographical expansion of visceral leishmaniasis \\ in the State of Pernambuco
}

\author{
Filipe Dantas-Torres ${ }^{1}$ e Sinval P. Brandão-Filho ${ }^{1}$
}

\begin{abstract}
RESUMO
Este estudo visa demonstrar a expansão geográfica da leishmaniose visceral no Estado de Pernambuco, Brasil. A partir do número de casos notificados de 1990 a 2001, foram elaborados mapas temáticos sobre a evolução bi-anual da distribuição geográfica da leishmaniose visceral por município. Por fim, foi construído um mapa da distribuição cumulativa dos casos resgistrados durante o período estudado. De 1990 a 2001, foram notificados 1.737 casos de leishmaniose visceral em Pernambuco. Em 1990, apenas 15,2\% $(n=28)$ dos municípios haviam registrado casos, enquanto, após pouco mais de uma década, esse percentual elevou-se para $78,3 \%(n=144)$. A distribuição geográfica cumulativa demonstra uma clara concentração de casos no Agreste e no Sertão. Houve uma considerável expansão geográfica da leishmaniose visceral em Pernambuco, refletindo, provavelmente, o baixo impacto das atuais medidas de controle e é possível que se deva também à melhoria do sistema de notificação.
\end{abstract}

Palavras-chaves: Leishmaniose visceral. Distribuição. Urbanização. Epidemiologia.

\begin{abstract}
This study aimed to demonstrate the geographical expansion of visceral leishmaniasis in Pernambuco State, Brazil. With data on the number of visceral leishmaniasis cases notified from 1990 to 2001, maps showing the biannual evolution of the geographical distribution of the disease per municipality were elaborated. A map of the cumulative geographical distribution of the cases registered during the whole period was also constructed. From 1990 to 2001, 1,737 cases of visceral leishmaniasis were notified in Pernambuco. In 1990, 15.2\% $(n=28)$ of the municipalities notified cases of the disease. In contrast, this percentage increased to $78.3 \%(n=144)$, over an eleven-year period. The map of cumulative geographical distribution during the whole period shows a notable concentration of cases in Agreste and Sertão. A notable geographical expansion of visceral leishmaniasis in Pernambuco also occurred, probably reflecting the low impact of the current control measures and, possibly, an improvement in the notification system.
\end{abstract}

Key-words: Visceral leishmaniasis. Distribution. Urbanization. Epidemiology.

No Brasil, a leishmaniose visceral (LV) apresenta uma ampla distribuição geográfica, além de alta letalidade, quando não instituído o tratamento adequado em tempo hábil. Associado ao seu espectro de morbidade, esta zoonose é causada por um protozoário de ciclo biológico complexo, o que a torna uma enfermidade de grande magnitude e de baixa vulnerabilidade às atuais medidas de controle. A escassez de recursos e a atual falta de infra-estrutura dos serviços de saúde, especialmente no que concerne ao diagnóstico da infecção por Leishmania chagasi (Kinetoplastida: Trypanosomatidae), na população canina e humana, tornam as atuais medidas de controle pouco factíveis. Esse quadro vem se constituindo como um paradigma, favorecendo a perpetuação do ciclo vicioso entre pobreza e doença em muitos estados brasileiros, nos quais a LV permanece como mais uma doença negligenciada. Em resposta a este cenário desfavorável, têm sido empreendidos vários esforços na tentativa de definir uma nova abordagem mais efetiva para o controle da doença no Brasil ${ }^{6}$.

\footnotetext{
1. Centro de Pesquisas Aggeu Magalhães da Fundação Oswaldo Cruz, Recife, PE, Brasil.

Endereço para correspondência: Dr. Sinval Pinto Brandão-Filho. CPqAM/FIOCRUZ, Campus UFPE, Av Moraes Rego s/n, $50670-420$ Recife, PE, Brasil.

Tel: 5581 2101-2562; Fax: 5581 3453-2449

e-mail: sinval@cpqam.fiocruz.br

Recebido para publicação em 19/9/2005

Aceito em 15/5/2006
} 
Atualmente, a LV é considerada endêmica em 19 estados do país, destacando-se aqueles da região Nordeste, responsáveis pela maior parte dos casos notificados ${ }^{12}$. Sobretudo nos últimos 20 anos, a doença difundiu-se e tornou-se cada vez mais comum em áreas urbanas ou periurbanas ${ }^{5131420}$. No Estado de Pernambuco, a situação não parece ser diferente. 0 paradigma da endemia rural é substituído pelo da doença associada a modificações ambientais, à ocupação desordenada do espaço urbano e às precárias condições de vida da população exposta ao risco. Logo, seja no espaço rural ou urbano, a LV amplia sua área de ocorrência, ultrapassando antigos limites geográficos definidos e tornando-se um sério problema de saúde pública em praticamente todo território pernambucano.

Assim, o objetivo do presente estudo é demonstrar a evolução da distribuição geográfica da LV no Estado de Pernambuco.

\section{MATERIAL E MÉTODOS}

Área de estudo. 0 Estado de Pernambuco está localizado no centro-leste da região Nordeste do Brasil, possui um território de $98.938 \mathrm{~km}^{2}$ e é constituído por 184 municípios, somando-se a esses o arquipélago de Fernando de Noronha, os quais estão distribuídos em cinco mesorregiões geográficas: Região Metropolitana de Recife, Zona da Mata, Agreste, Sertão e Sertão do São Francisco (Figura 1). Com uma população de 7.918.344 habitantes, Pernambuco tem passado por um intenso processo de urbanização, claramente refletido na representatividade de sua população urbana, 6.058.249 habitantes, que em 2000 correspondia a 76,5\% da população total.
Fonte de dados e construção dos mapas temáticos. Os dados referentes aos casos de $\mathrm{LV}$, notificados no período de 1990 a 2001, foram fornecidos pela Secretaria Estadual de Saúde de Pernambuco (SES-PE). A partir do valor bruto do total de casos notificados, foram construídos mapas temáticos que demonstram a evolução bi-anual da distribuição geográfica da LV por município. Também, foi elaborado um mapa da distribuição geográfica cumulativa dos casos notificados durante todo o período de estudo (1990-2001). Para construção dos mapas temáticos, foi utilizado o programa TerraView (release 3.0.3, INPE, Brasil), tomando-se como base cartográfica o mapa de Pernambuco, disponibilizado pelo Instituto Brasileiro de Geografia e Estatística (IBGE).

\section{RESULTADOS}

De acordo com os dados da SES-PE, foram notificados 1.737 casos de LV em Pernambuco, entre 1990 e 2001. No início do período estudado (1990), apenas 15,2\% $(n=28)$ dos municípios do estado haviam notificado um ou mais casos da doença. Ao final do período (1990-2001), esse percentual elevou-se para 78,3\% $(n=144)$. Os municípios com maior número de casos notificados, ao longo do período estudado, encontram-se listados na Tabela 1.

A Figura 2 representa a evolução bi-anual da distribuição geográfica dos casos de LV notificados em Pernambuco. De 1990 a 1991 (Figura 2A), houve um predomínio de casos no Sertão e no Agreste, havendo, contudo, registro de casos em outras regiões, notadamente na Região Metropolitana do Recife. Nos dois anos seguintes (Figura 2B), o quadro não foi

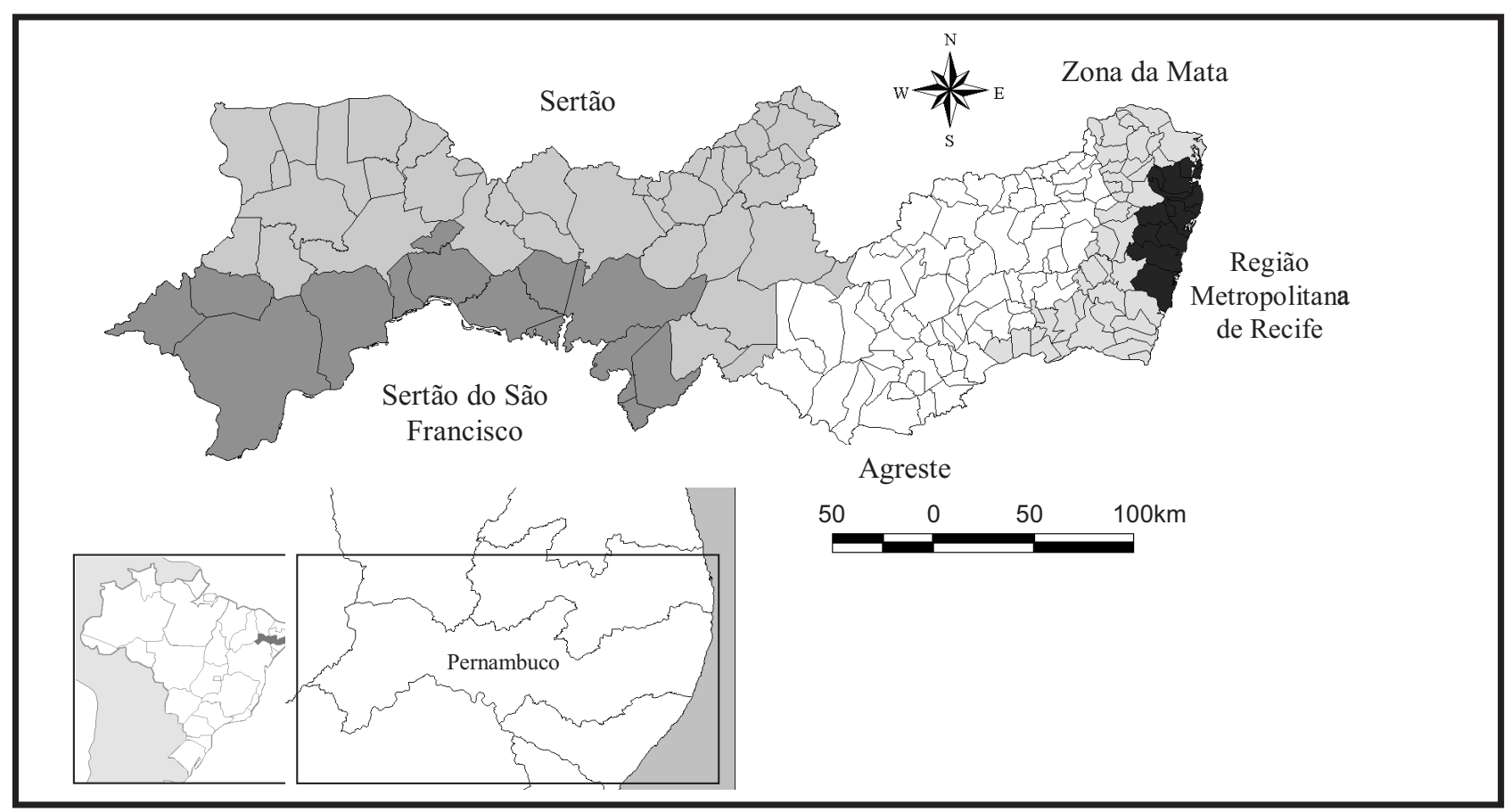

Figura 1 - Regiões geográficas do Estado de Pernambuco, Brasil. 
muito diferente, observando-se apenas uma discreta redução do número de casos registrados no estado como um todo. Já no período de 1994 a 1995 (Figura 2C), ocorreu uma situação bem diferente: 0 número de casos notificados nesse período supera o quantitativo registrado nos últimos quatro anos. Houve um aumento importante do número de casos em todas as regiões de Pernambuco, mormente no Agreste. No período de 1996 a 1997 (Figura 2D), houve uma nova redução do número de casos notificados no estado, permanecendo, contudo, uma discreta concentração de casos no Agreste. Esta concentração acentuouse no período de 1998 a 1999 (Figura 2E), com a formação de um cluster em volta do município de Caruaru. Nos dois últimos anos do período estudado (Figura 2F), ocorreu um notável aumento do número de casos notificados, em todas as regiões de Pernambuco, com a re-emergência de antigos focos da doença, assim como emergência de novos, a exemplo do município de Petrolina, localizado no Sertão do São Francisco.

0 mapa da distribuição geográfica cumulativa dos casos de $\mathrm{LV}$ notificados em Pernambuco, durante todo o período estudado (Figura 3), demonstra uma clara concentração de casos em municípios do Agreste e do Sertão. Entretanto, alguns municípios de outras regiões, tais como Itamaracá na Região Metropolitana do Recife, destacaram-se entre aqueles que mais notificaram casos, ao longo do período estudado.
Tabela 1 - Municípios que mais notificaram casos de leishmaniose visceral em Pernambuco, Brasil, 1990-2001.

\begin{tabular}{|c|c|c|}
\hline \multirow[b]{2}{*}{ Município } & \multicolumn{2}{|c|}{ Casos notificados } \\
\hline & $\mathrm{n}^{0}$ & $\%$ \\
\hline Caruaru & 165 & 17,2 \\
\hline Ilha de Itamaracá & 102 & 10,6 \\
\hline Salgueiro & 74 & 7,7 \\
\hline Altinho & 65 & 6,8 \\
\hline Surubim & 63 & 6,5 \\
\hline São Caitano & 51 & 5,3 \\
\hline Goiana & 44 & 4,6 \\
\hline São Vicente Ferrer & 42 & 4,4 \\
\hline Petrolina & 42 & 4,4 \\
\hline Riacho das Almas & 38 & 4,0 \\
\hline Serra Talhada & 35 & 3,6 \\
\hline São Bento do Una & 31 & 3,2 \\
\hline Paulista & 30 & 3,1 \\
\hline Vertentes & 28 & 2,9 \\
\hline Taquaritinga do Norte & 27 & 2,8 \\
\hline Frei Miguelinho & 27 & 2,8 \\
\hline Agrestina & 25 & 2,6 \\
\hline Cachoeirinha & 25 & 2,6 \\
\hline Glória do Goitá & 25 & 2,6 \\
\hline Águas Belas & 23 & 2,4 \\
\hline Total & 962 & 100,0 \\
\hline
\end{tabular}

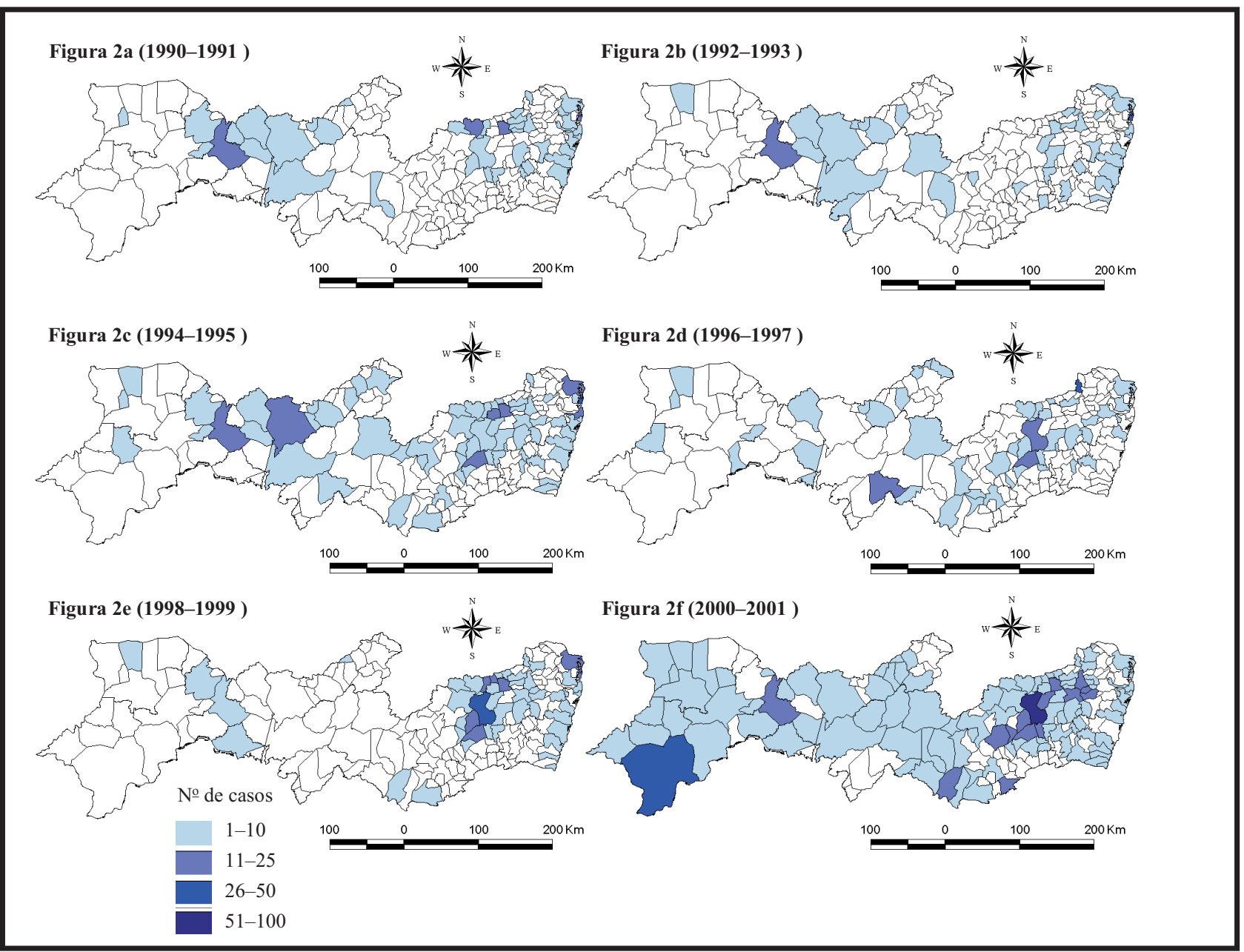

Figura 2 - Evolução bi-anual da distribuição geográfica dos casos de leishmaniose visceral notificados em Pernambuco, Brasil, 1990 -2001. 


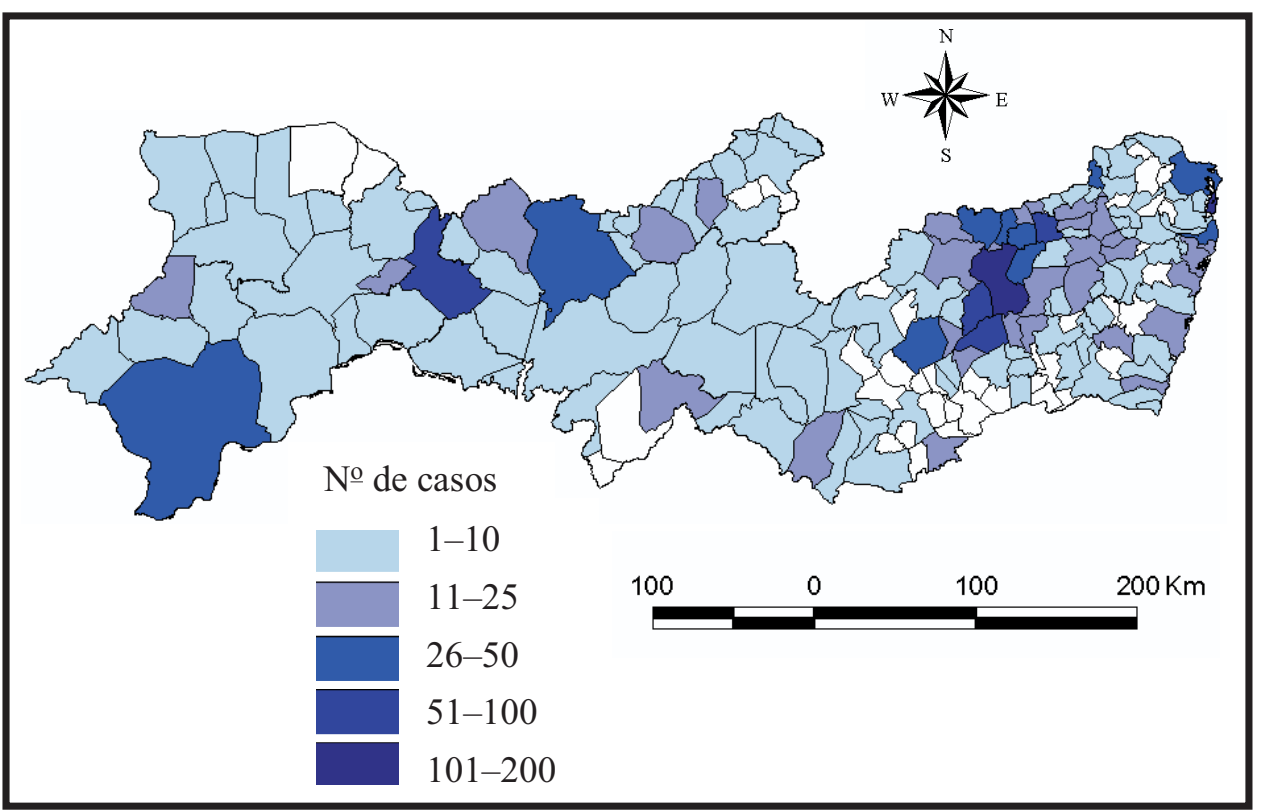

Figura 3 - Distribuição geográfica cumulativa dos casos de leishmaniose visceral notificados em Pernambuco, Brasil, 1990-2001.

\section{DISCUSSÃo}

O estudo da distribuição geográfica, seja ele analítico ou essencialmente descritivo, tem sido ferramenta largamente utilizada em estudos epidemiológicos, inclusive, relacionados à LV 23161721 . Esses estudos, notadamente quando são aplicadas técnicas de análise espacial, permitem identificar padrões espaciais de morbidade e/ou mortalidade e possíveis fatores associados, bem como descrever o processo de difusão das doenças, gerando informações sobre a gênese dos agravos que acometem determinadas populações, objetivando sua predição, prevenção e controle ${ }^{15}$. 0 presente estudo demonstra que houve uma acentuada expansão geográfica da LV em Pernambuco, entre 1990 e 2001. Além do surgimento de novos focos, foi observada a persistência das antigas áreas de ocorrência da doença. Isso demonstra, no mínimo, que as atuais medidas de controle estão sendo insuficientes, seja para controlar a LV nas áreas endêmicas, seja para prevenir a ativação, ou reativação, de focos em áreas até então consideradas indenes.

No período estudado, a LV difundiu-se não só nas áreas clássicas do Agreste e Sertão, onde está concentrado um número importante de casos, mas também em outras regiões, notadamente na Região Metropolitana de Recife. Diversos fatores podem ter contribuído para a disseminação da doença em Pernambuco. Dentre estes, podemos destacar o intenso fluxo migratório intermunicipal, sobretudo do interior do estado para a Região Metropolitana de Recife, mas também no sentido inverso. Estes movimentos populacionais permitem tanto a introdução do agente causador da LV em áreas livres, quanto à inserção de indíviduos susceptíveis em áreas endêmicas. Muitas famílias vindas do interior se estabelecem na periferia das cidades de médio e grande porte, formando aglomerados densamente povoados que apresentam precárias condições de infra-estrutura e saneamento básico. Com efeito, observa-se a presença de crianças que, em geral, devido à seca que devasta o interior do estado, periodicamente, apresentam-se mal nutridas, condição esta que favorece sobremaneira a expressão e 0 agravamento da LV. Já foi previamente demonstrado que, nestas condições, com a presença de um grande contingente de hospedeiros susceptíveis, a doença pode assumir caráter epidêmico ${ }^{15713}$.

Certamente, outros fatores devem ter influenciado o processo de expansão geográfica da LV em Pernambuco. Em Petrolina, foi demonstrado que a organização do espaço urbano pode influenciar enormemente a expansão da doença ${ }^{4}$. No Estado de Pernambuco, de fato, a maioria dos casos parece está associada a pressão antrópica sobre 0 ambiente e ocupação desordenada do espaço físico ${ }^{19}$. Outro aspecto importante a ser considerado é a notável capacidade de domiciliação do flebotomíneo Lutzomyia longipalpis (Diptera: Psychodidae), o qual atualmente pode ser encontrado em praticamente todo território pernambucano.

Como já demonstrado em outros estados, a distribuição geográfica da LV em Pernambuco reforça a superação do paradigma da doença tipicamente rural. Neste estado, o ciclo zoonótico da LV encontra-se claramente estabelecido em áreas urbanas e periurbanas, como nos municípios de Petrolina ${ }^{4}$ e Paulista ${ }^{11}$. Contudo, nas áreas rurais, onde as precárias condições de vida da população são importantes determinantes do processo saúde-doença, assim como em áreas urbanas, a LV permanece como uma doença negligenciada, acometendo principalmente crianças ${ }^{19}$, sobretudo as mal nutridas ${ }^{18}$.

Devemos destacar algumas limitações observadas no presente estudo. Como já foi dito, os casos aqui incluídos correspondem a dados secundários da SES-PE. Observa-se em Pernambuco, de forma geral, algumas dificuldades em relação ao diagnóstico da LV. Portanto, é possível que, ao longo do período estudado, alguns casos não tenham sido notificados por falta de diagnóstico e, conseqüentemente, a real magnitude do problema tenha sido subestimada. Além disso, foram incluídos casos registrados 
em municípios como Recife, onde a presença de Lutzomyia longipalpis ainda não foi claramente demonstrada, sendo estes certamente alóctones ${ }^{89}{ }^{10}$. É preciso lembrar ainda que, uma vez que a população dos municípios pode variar enormemente, o valor bruto do total de casos apenas nos informa quais municípios notificaram, não sendo suficiente para indicar se houve maior ou menor risco à infecção e ao adoecimento em cada município.

Apesar das eventuais limitações, o presente trabalho demonstra categoricamente uma acentuada expansão geográfica da LV em Pernambuco. Serve ainda de alerta para a necessidade de novas investigações sobre o tema, com ênfase na definição das áreas de risco e na avaliação do real impacto das atuais estratégias de controle sobre a incidência da doença na população.

\section{AGRADECIMENTOS}

À Secretaria Estadual de Saúde de Pernambuco, por ceder gentilmente os dados e pelo apoio logístico que tem prestado ao nosso grupo do Centro de Pesquisas Aggeu Magalhães/ FIOCRUZ, durante as pesquisas sobre as leishmanioses em Pernambuco. Ao Dr. Wayner Vieira de Souza pelas opiniões e a José Constantino Silveira Júnior pelo apoio na elaboração dos mapas temáticos.

\section{REFERÊNCIAS BIBLIOGRÁFICAS}

1. Arias JR, Monteiro PS, Zicker F. The reemergence of visceral leishmaniasis in Brazil. Emerging Infectious Diseases 2:145-146, 1996.

2. Bevilacqua PD, Paixão HH, Modena CM, Castro MCPS. Urbanização da leishmaniose visceral em Belo Horizonte. Arquivos Brasileiros de Medicina Veterinária e Zootecnia 53:1-8, 2001.

3. Camargo-Neves VLF, Katz G, Rodas LAC, Poletto DW, Lage LC, Spínola RMF, Cruz OG. Utilização de ferramentas de análise espacial na vigilância epidemiológica de leishmaniose visceral americana - Araçatuba, São Paulo, Brasil, 1998-1999. Cadernos de Saúde Pública 17:1263-1267, 2001.

4. Cesse EAP, Carvalho EF, Andrade PP, Ramalho WM, Luna L. Organização do espaço urbano e expansão do calazar. Revista Brasileira de Saúde Materna e Infantil 1:167-176, 2001

5. Costa CHN, Pereira HF, Araújo MV. Epidemia de leishmaniose visceral no estado do Piauí, Brasil, 1980-1986. Revista de Saúde Pública 24:361-372, 1990

6. Costa CHN, Vieira JBF. Mudanças no controle da leishmaniose visceral no Brasil. Revista da Sociedade Brasileira de Medicina Tropical 34:223-228, 2001.
7. Costa JML, Viana GMC, Saldanha ACR, Nascimento MDSB, Alvim AC, Burattini MN, Silva AR. Leishmaniose visceral no estado do Maranhão, Brasil: a evolução de uma epidemia. Cadernos de Saúde Pública 11:321324, 1995.

8. Dantas-Torres F, Brandão-Filho SP. A leishmaniose visceral é uma doença endêmica no Recife, Pernambuco? Revista da Sociedade Brasileira de Medicina Tropical 38:361-362, 2005.

9. Dantas-Torres F, Faustino MAG, Alves LC, Acioli R, Lima OC. Classificação do município do Recife quanto à transmissão da leishmaniose visceral canina. Revista Brasileira de Parasitologia Veterinária 13 (supl I):234, 2004.

10. Dantas-Torres F, Faustino MAG, Lima OCC, Acioli RV. Epidemiologic surveillance of canine visceral leishmaniasis in the municipality of Recife, Pernambuco. Revista da Sociedade Brasileira de Medicina Tropical 38:444$445,2005$.

11. Dantas-Torres F, Francisco D, Brandão-Filho SP. Análise situacional da leishmaniose visceral no município de Paulista, Pernambuco, nordeste do Brasil. In: Resumos do I Congresso Norte-Nordeste de Zoonoses e Bem Estar Animal, Natal, 1 CD-ROM, 2004.

12. Gontijo CMF, Melo MN. Leishmaniose visceral no Brasil: quadro atual, desafios e perspectivas. Revista Brasileira de Epidemiologia 7:338-349, 2004 .

13. Jerônimo SMB, Oliveira RM, Mackay S, Costa RM, Sweet J, Nascimento ET, Luz KG, Fernandes MZ, Jernigan J, Pearson RD. An urban outbreak of visceral leishmaniasis in Natal, Brazil. Transactions of the Royal Society of Tropical Medicine and Hygiene 88:386-388, 1994.

14. Luz ZMP, Pimenta DN, Cabral AL, Fiuza VO, Rabello A. A urbanização das leishmanioses e a baixa resolutividade diagnóstica em municípios da Região Metropolitana de Belo Horizonte. Revista da Sociedade Brasileira de Medicina Tropical 34:249-254, 2001.

15. Medronho RA, Werneck GL. Técnicas de análise espacial em saúde In: Medronho RA (org) Epidemiologia. Atheneu, São Paulo p.427-446, 2004.

16. Mendes WS, Silva AAM, Trovão JR, Silva AR, Costa JML. Expansão espacial da leishmaniose visceral americana em São Luis, Maranhão, Brasil. Revista da Sociedade Brasileira de Medicina Tropical 35:227-231, 2002.

17. Oliveira CDL, Assunção RM, Reis IA, Proietti FA. Spatial distribution of human and canine visceral leishmaniasis in Belo Horizonte, Minas Gerais State, Brasil, 1994-1997. Cadernos de Saúde Pública 17:1231-1239, 2001.

18. Queiroz MJA, Alves JGB, Correia JB. Visceral leishmaniasis: clinical and epidemiological features of children in an endemic area. Jornal de Pediatria 80:141-146, 2004

19. Silva DF, Vasconcelos SD. A ten year (1990-1999) survey on leishmaniasis incidence in Pernambuco state, northeastern Brazil. Revista de Patologia Tropical 32:51-61, 2002

20. Silva ES, Gontijo CMF, Pacheco RS, Fiúza VOP, Brazil RP. Visceral leishmaniasis in the Metropolitan Region of Belo Horizonte, State of Minas Gerais, Brazil. Memórias do Instituto 0swaldo Cruz 96:285-291, 2001.

21. Werneck GL, Maguire JH. Spatial modeling using mixed models: an ecologic study of visceral leishmaniasis in Teresina, Piauí State, Brazil. Cadernos de Saúde Pública 18:633-637, 2002. 